\title{
Papulosquamous eruption with ocular symptoms caused by syphilis
}

\author{
Marcus G. Tan MD, Mark G. Kirchhof MD PhD
}

— Cite as: CMAJ 2020 December 7;192:E1727. doi: 10.1503/cmaj.200820

A n otherwise healthy 42-year-old woman presented to the emergency department with a 1-week history of subjective fever, worsening headache, blurry vision, photophobia, nausea and epiphora (Figure 1A). On physical examination, the patient was afebrile but had a widespread asymptomatic papulosquamous eruption (Figure 1B and C). The rash had been present for several weeks, but she had not previously sought medical attention because it was asymptomatic. Slit-lamp examination suggested anterior uveitis. Two months earlier, the patient had tested negative for syphilis, HIV, gonorrhea and chlamydia 1 week after unprotected sexual intercourse with a new partner.

An abdominal skin biopsy showed vacuolar interface changes with superficial and mid-dermal mixed inflammatory infiltrates of plasma cells, lymphocytes and histiocytes. Immunohistochemical stains showed spirochetes. Results of baseline blood work were unremarkable. A syphilis screen with chemiluminescent immunoassay and rapid plasma reagin was positive, as was confirmatory testing with Treponema pallidum particulate agglutination assay.

We diagnosed secondary syphilis with ocular neurosyphilis. Because of the patient's penicillin allergy, we prescribed a 28-day course of oral doxycycline (100 mg twice daily). Her constitutional and ocular symptoms resolved within 1 week, and her rash was almost completely resolved in 1 month.

Clinicians should maintain a high degree of suspicion for secondary syphilis in patients presenting with widespread papulosquamous eruptions. When secondary syphilis is suspected, clinicians should repeat syphilis testing, even if recent screening results were negative. ${ }^{1}$ In early primary syphilis, false-negative test results occur because seroconversion requires 14-21 days. False-negative results can also occur because of the "prozone phenomenon," where high antibody titres interfere with the formation of antibody-antigen complexes that are necessary to induce a positive test result. ${ }^{2}$ Although neurosyphilis is often regarded as a form of tertiary syphilis, it can manifest at any stage of the disease. ${ }^{3}$ Follow-up rapid plasma reagin tests should be performed at 6 months and 12 months after treatment to ensure complete response, which is indicated by a fourfold decline in titres.

\section{References}

1. Li L, Cai B, Tao C, et al. Performance evaluation of CLIA for Treponema Pallidum specific antibodies detection in comparison with ELISA. J Clin Lab Anal 2016;30:216-22.

2. Ghanem KG, Ram S, Rice PA. The modern epidemic of syphilis. N Engl J Med 2020;382:845-54.

3. Quilter LAS, de Voux A, Amiya RM, et al. Prevalence of self-reported neurologic and ocular symptoms in early syphilis cases. Clin Infect Dis [Epub ahead of print] 2020 Feb 26;ciaa180. doi: 10.1093/cid/ciaa180.

\section{Competing interests: None declared.}

This article has been peer reviewed.

The authors have obtained patient consent.

Affiliations: Division of Dermatology, Faculty of Medicine, University of Ottawa; The Ottawa Hospital, Ottawa, Ont.

Correspondence to: Mark Kirchhof, mkirchho@uottawa.ca 